\title{
METHODOLOGICAL FOUNDATIONS OF MHEALTH DESIGN STUDIES: A CONTENT ANALYSIS OF LITERATURE
}

\author{
Ahmet Faruk Acar and Sevgi Özkan Yıldırım \\ METU \\ Ankara, Turkey
}

\begin{abstract}
Despite the pervasiveness of mobile devices and the number of available mobile health (mHealth) apps, a scaled nationwide mHealth solution for supporting continuous healthcare is lacking in many developing countries. Systematic reviews in mobile health field show various barriers to explain the lack of scalability and sustainability among mHealth interventions. However, little is known about the methodological soundness of mHealth app design studies. We conducted a literature review to investigate the theoretical foundations of mHealth design studies, which are essential to inform design and content of the solution. The purpose of this paper is to evaluate and classify the mHealth studies in terms of their methodological soundness for the design, development process of smartphone application (app) through content analysis.
\end{abstract}

\section{KEYWORDS}

mHealth, Methodology, Design, Development, Evidence-Based

\section{INTRODUCTION}

mHealth stands out as a promising intervention method to provide medical consultancy, social support, and evidence-based information. Despite the obvious need and benefits of mHealth systems for self-management, behavior change and provider consultancy; sustainable, scaled and well-adopted mHealth systems are lacking in many developing counties. Literature review studies brings different aspects regarding the barriers and facilitators towards design, development and implementation of mHealth applications (app) such as financial issues, policies, privacy and security concerns, individual and organizational readiness.

Alvarado et al. categorize the barriers of remote health interventions as patient barriers, technology access barriers, design barriers, provider barriers, and system barriers (Alvarado et al., 2017). According to review of Matthew-Maich et al., organizational and system readiness, financial resources, policies, and workplace culture all play a key role in the successful adoption of mHealth technologies. Also their review refers to the inertia and resistance to change that can exist within organizations (Matthew-Maich et al., 2016). Kim et al. also refer to organizational readiness by the fact that current healthcare systems are not adequately designed to meet the long-term management needs of individuals, instead, suited better for the acute management (Kim et al., 2017).

Factors such as the cost of implementing the system, increased clinical workload and workflow, maintaining up-to-date records, and concerns about being supervised and depending too much on technology are also given as the main concerns regarding implementation of mHealth adherence interventions (Hamine et al., 2015). Steinhuble et al. emphasize the current fee-for-service reimbursement model in healthcare as a major financial barrier due to lack of financial incentive for practitioners to implement such mHealth systems. They propose value-based care instead of fee-for-service but the former is not likely to replace the other very soon (Steinhubl et al., 2015).

The mHealth field is relatively new and the main assumption of this paper is that mHealth studies may lack the methodological rigor. Therefore, mHealth studies need further investigation in terms of their theoretical and methodological soundness besides the various aspects given in the reviews above. In this 
study, we conducted a literature review and analyzed mHealth studies in terms of theoretical and other systematic approaches that substantiates their design, development and implementation methods. Our content analysis shows that mHealth studies may have evidence-based and/or design-based theoretical basis while some of them possess none. Following sections have the details of literature review's results.

\section{METHODOLOGY}

We conducted a literature review to find mHealth studies, which has at least one of design, development and implementation phases of an mHealth application. The key eligibility criteria were a smartphone application (or at least a prototype with high or low fidelity) should be developed within the scope of study. Furthermore, we aim only for peer-reviewed journal articles which are expected to have more rigorous methodology for the design, development phases and exclude review studies, conference papers and other studies from grey literature. Smartphone applications become ubiquitous in the last 10 years and even mobile phones were common before 2009, mHealth studies mostly focused on SMS and text-based intervention and lacked the design, development process of smartphone apps and complex interaction of its users. Therefore, journal articles which are published in the last 10 years are particularly sought for.

\subsection{Identification}

Medline, Scopus and Web of Science are chosen as scientific databases to have optimum coverage for our search results. Keywords and Boolean representation of the search query is formulated (Table 1) and run within the title field of studies that are published in the last 10 years.

Table 1. Keywords and boolean representation of search query

\begin{tabular}{ll}
\hline Keywords & Boolean Representation \\
\hline design, development, implementation, mhealth, & ((design OR development OR implementation) AND (mhealth OR \\
mobile health, m-health, mobile app, mobile & mobile health OR m-health OR mobile app OR mobile application \\
application, smartphone application & OR smartphone application)) \\
\hline
\end{tabular}

List of the search criteria used for all three digital libraries are as follows:

- Publication date is between '2009/01/01 - 2019/01/01'

- Publication type is 'Journal Article'

- Publication language is 'English'

- Full text and abstract is available

Medline, Scopus and Web of Science database searches yield 306, 100 and 241 results respectively. After the duplications were removed, 429 articles left for screening. Based on the PRISMA guidelines (Moher, 2009), literature review is conducted as in the following section.

\section{RESULTS}

\subsection{Screening}

The researcher (corresponding author of this paper) screened the titles and abstracts of 429 articles to check the focus of the studies and filtered down the related ones based on the eligibility criteria. Author names and publication year of the articles are hidden during this procedure to prevent bias. Articles which are excluded from final evaluation can be described as:

- Purely social or psychological studies which doesn't have mobile application design phase

- Technical studies that focus on only algorithm or hardware design for mHealth

- Literature review studies 
- Study protocols which describes how the mHealth design will be conducted but doesn't involve the actual design and development process.

- Studies that use an existing mobile health app for evaluation but doesn't involve any of the design, development and implementation phases

- Qualitative studies which only does requirement elicitation for mHealth but doesn't involve any of the design, development and implementation phases

- mHealth studies which focus solely on content/text/message design

Screening phase yields 123 articles for eligibility.

\subsection{Eligibility}

Relevant 123 journal articles are reviewed and analyzed by the researcher for their theoretical soundness and methodological rigor. Analysis is done in an iterative fashion. In the first iteration, the researcher read the 123 articles and write keywords about the frameworks, methodological approaches, tools and theoretical basis that substantiate the design, development phases of each mHealth study such as "focus-group discussion", "interdisciplinary team approach", "Theory of Planned Behavior (TPB)", "use of clinical guidelines", "workshops", "agile development", "pilot phase" and "interview" etc. Accumulated list of keywords was then used to group articles into the following classifications for their methodological approach:

- 'Design-based studies' is used to annotate articles which comprise theories and frameworks that are mostly originated from software engineering or other Information Systems (IS) related fields to inform the design, development and implementation processes of mHealth study

- 'Evidence-based studies' is used to annotate articles which comprise theories, existing behavior change techniques (BCTs), and guidelines that is mostly rooted in behavioral, clinical science to inform the content and intervention components of mHealth study

- 'None' is used to annotate articles which doesn't have any theoretical basis or systematic approach to design, development and implementation of their mHealth app design

\subsection{Included}

In the second iteration, 123 articles were coded with one of these three codes. Consequently, 47 (39\%) studies are coded with 'None' which means they lack any kind of systematic approach for the design phase and they also lack refer to evidence from literature to initiate the content for the mHealth app they developed. Remaining 76 (61\%) is coded with one or two of the codes and only 12 (9\%) studies reports the use of both evidence-based and design-based approaches in their methodology. Also, 35 (28\%) articles adopts design-based approach without referring to evidence-based and 29 (23\%) articles comprise only evidence-based information without any design-based approach. Details of the results and implications are discussed in the following section.

\section{DISCUSSION}

\subsection{Design-based mHealth Studies}

Among the 123 articles, 35 (28\%) studies adopt at least one systematic approach to inform the design stage of mHealth app. Design-based frameworks commonly have roots in software engineering or other Information Systems (IS) related fields. List of frameworks and theories on which mHealth app design studies grounded is given in Table 2. 
Table 2. Frameworks and Theories that inform the mHealth Studies Design

\begin{tabular}{lc}
\hline Theory \& Framework & \# of Study \\
\hline User-center design & $\mathbf{1 8}$ \\
\hline Participatory design & $\mathbf{5}$ \\
\hline Analysis Design Development Implementation Evaluation (ADDIE) & $\mathbf{4}$ \\
\hline Design Science Research & $\mathbf{3}$ \\
\hline Medical Research Council (MRC) Framework & $\mathbf{3}$ \\
\hline Web-Roadmap Methodology & $\mathbf{1}$ \\
\hline Formative Evaluation Framework & $\mathbf{1}$ \\
\hline Validated Learning & $\mathbf{1}$ \\
\hline Grounded Theory & $\mathbf{1}$ \\
\hline ICT Implementation Framework & $\mathbf{1}$ \\
\hline Consolidated Framework for Implementation Research & $\mathbf{1}$ \\
\hline IDEAS Framework & $\mathbf{1}$ \\
\hline Rapid Application Development (RAD) Framework & $\mathbf{1}$ \\
\hline Waterfall Model & $\mathbf{1}$ \\
\hline
\end{tabular}

Not all the frameworks and theories mutually exclusive in their use, since some of the studies prefer to use more than one approach in their study. User-centered design (UCD) is the most common approach for mHealth app design process. Eighteen articles report the use of UCD principles, methods and materials such as focus-group discussions, interviews, and surveys. Even UCD approach provides certain principles for the involvement of end users in the design process, it still lacks the systematic approach to structure overall study or evaluate the mHealth intervention besides usability. However, usability is merely one of many concerns for mHealth design, development and evaluation. For instance, (Goyal et al., 2016) follows the literature research, iterative developing and piloting phases of the of the Medical Research Council's (MRC) model along with UCD methods to assist with the development and evaluation of health technology interventions.

Participatory action research design is the second most common approach among the design-based studies. Involvement of stakeholders during workshops and focus-groups are common methods used during the design, development of mHealth apps. Having interdisciplinary teams, which consists of professionals from technical, clinical and organizational backgrounds for the co-design efforts is the key feature of the participatory design research (Ravn et al., 2018; Revenäs et al., 2015). Lin et al., which also adopts participatory design, highlights the importance of adaptive intervention design (AID) approach to be able to update an intervention throughout a trial while assuring the delivery of the same intervention to every participant (Lin et al., 2015).

Four studies use the standard life cycle of system development steps: analysis, design, implementation, and evaluation (ADDIE) to structure their mHealth design phase. López et al. use ADDIE steps to create their "MyNutriCart" app to improve food selection in the grocery stores based on dietary guidelines (López et al., 2017). In Analysis step, studies extracted system requirements from various resources such as literature review (López et al., 2017), clinical guidelines (Jeon and Park, 2015; Jo and Park, 2016) but none of these studies involve end users into Analysis step.

Software development frameworks such as ICT Implementation Framework and Waterfall model inform the design and development of mHealth studies (Mehmood et al., 2017; Thinnukool et al., 2017). Additionally, the study of Ithnin et al., adopts an agile methodology for the design and development phases (Ithnin et al., 2017).

Only three articles refer to Design Science Research (DSR) as a comprehensive framework to structure their design process. Schnall et al. employs the framework as an overarching theoretical foundation of the mHealth study (Schnall et al., 2016). Based on DSR framework's 3 cycles: they conducted focus groups with end-users in the Relevance cycle; performed a review to identify technology-based interventions for meeting the health prevention needs in the Rigor cycle and they employed usability evaluation methods to iteratively develop and refine mock-ups for a mHealth app in the Design Cycle. Alharbi et al and Alnosayan et al also align their design methodology with the DSR framework's three cycles (Alharbi et al., 2018; Alnosayan et al., 2017). 


\subsection{Evidence-based mHealth Studies}

Thirty-one (24\%) of 123 studies report at least one evidence-based method such as BCT and guidelines for determining content and components of mHealth intervention. Evidence-based approaches are mostly derived from one of Behavior Change Techniques, clinical guidelines and some studies create their own customized technique by blending several BCTs and guidelines to achieve optimized content, algorithm, and intervention components. The list of the BCTs and guidelines used for evidence-based mHealth studies is given in Table 3 .

Table 3. Evidence-based Theories and Guidelines

\begin{tabular}{|c|c|c|}
\hline BCT/Guideline & $\#$ & Name \\
\hline $\begin{array}{l}\text { Behavior } \\
\text { Change } \\
\text { Techniques } \\
\text { (BCTs) }\end{array}$ & 35 & $\begin{array}{l}\text { Social Cognitive Theory (SCT), Self-determination Theory (SDT), Information-Motivation- } \\
\text { Behavioral Skills Model, Theory of Planned Behavior (TPB), Cues to Action, The } \\
\text { Cognitive-Behavioral Model, Stress-vulnerability Model, The Early Intervention Model, Brief } \\
\text { Behavioral Activation Treatment for Depression (BATD), Self-regulation Model, Strength } \\
\text { Model of Self-control, Self-efficacy Theory, Social Exchange Theory, Motivational Interviewing } \\
\text { (MI) principles, Dietary Approaches to Stop Hypertension (DASH), Persuasive Techniques, } \\
\text { Behavioral Activation Treatment, Goal Setting Theory, The Knowledge to Action (KTA)... }\end{array}$ \\
\hline Guidelines & 6 & $\begin{array}{l}\text { CAIDE Dementia Risk Score, Swedish Web-based Quality of Recovery (SwQoR) questionnaire, } \\
\text { Food Pyramid and Mediterranean Diet guidelines, Dietary Guidelines for Americans... }\end{array}$ \\
\hline
\end{tabular}

Social Cognitive Theory (SCT) is the most common theory among behavioral change techniques to inform the mHealth intervention. For example, Choo et al. derived theoretical basis for their mHealth study from SCT by adopting key mediators such as; self-regulation skills, goal setting and self-monitoring of behaviors (Choo et al., 2016). Other studies (Lubans et al., 2014; Zhu et al., 2017; Lau et al., 2018) create their own blended theoretical basis by making use of multiple frameworks. In addition, six studies use guidelines to create the intervention component or algorithm of the mHealth intervention (Gabrielli et al., 2017; López et al., 2017).

\subsection{Other Studies and Descriptive Statistics}

While 47 studies (39\%) did not mention any systematic approach for the design phase or ground their intervention development with evidence-base, only $12(9 \%)$ studies adopted both evidence-based and systematic design approaches. Overall distribution of the articles according to years is given in Error! Reference source not found.

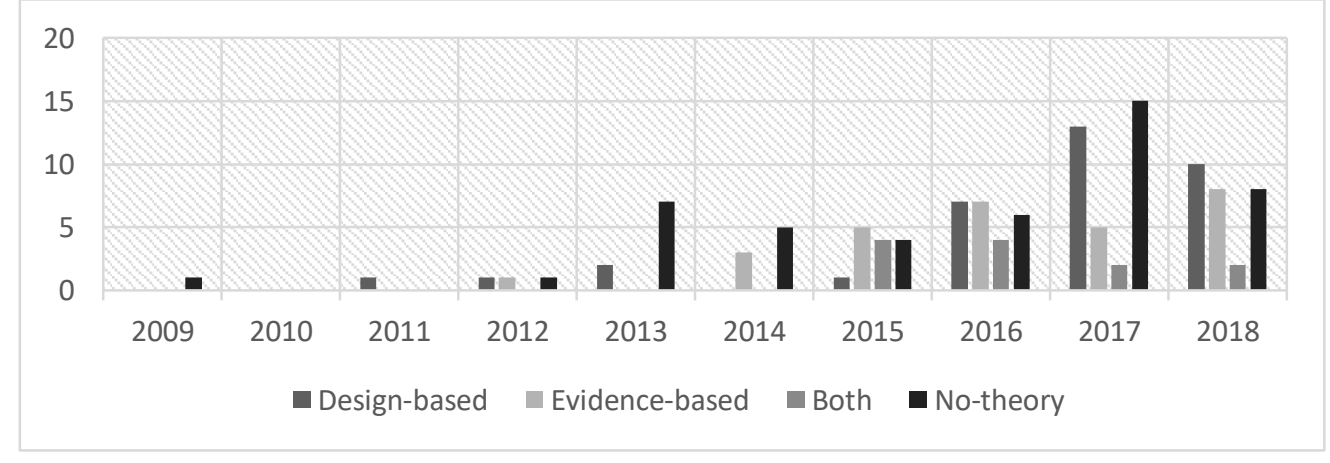

Figure 1. Distribution of mHealth studies based on the existence of theoretical approaches

Total number of mHealth studies that comprise design and development phases of a smartphone application is increasing since 2008. Only after 2012, design-based and evidence-based studies have emerged and upsurge in numbers. However, mHealth design studies without any theoretical foundations or systematics approaches have risen in numbers too. This recent rise in the number of publications are 
indicators for a need of rigorous methodology for future mHealth studies. The studies, which have both design-based and evidence-based techniques in their methodology are summarized in Table 4.

Table 4 Summary of mHealth studies that have both design-based and evidence-based methodology

\begin{tabular}{|c|c|c|}
\hline \multirow[t]{2}{*}{ Author, Year } & \multicolumn{2}{|c|}{ Methodology } \\
\hline & Design-based & Evidence-based \\
\hline $\begin{array}{l}\text { (Jeon and Park, } \\
\text { 2015) }\end{array}$ & $\begin{array}{l}\text { The study was conducted in the order of } \\
\text { analysis-design-implementation-evaluation } \\
\text { (ADDIE) according to the standard lifecycle } \\
\text { of system development }\end{array}$ & $\begin{array}{l}\text { Obesity-related knowledge and functional requirements } \\
\text { were extracted from clinical practice guidelines. }\end{array}$ \\
\hline $\begin{array}{l}\text { (Lin et al., } \\
2015)\end{array}$ & $\begin{array}{l}\text { Initial intervention development utilized focus } \\
\text { groups and iterative, participatory design. } \\
\text { Intervention testing employed adaptive } \\
\text { intervention design. }\end{array}$ & $\begin{array}{l}\text { Active interventions are designed based on social } \\
\text { cognitive theory and incorporated techniques. }\end{array}$ \\
\hline $\begin{array}{l}\text { (Revenäs } \text { et } \\
\text { al., 2015) }\end{array}$ & $\begin{array}{l}\text { Qualitative data is collected using multiple } \\
\text { methods in four workshops via participatory } \\
\text { design. }\end{array}$ & $\begin{array}{l}\text { Correlations between the system requirements and the } \\
\text { established behavior change techniques (BCTs) is } \\
\text { explored. }\end{array}$ \\
\hline $\begin{array}{l}\text { (Tregarthen } e t \\
\text { al., 2015) }\end{array}$ & $\begin{array}{l}\text { Validated learning is established through two } \\
\text { processes: inclusion of feedback fields into the } \\
\text { app and measuring utilization metrics to assess } \\
\text { the additive value of new features. }\end{array}$ & $\begin{array}{l}\text { The concept of the application is defined with the } \\
\text { intention to target aspects of Cognitive Behavioral } \\
\text { Therapy (CBT) treatment that require behavioral } \\
\text { activation and assimilation into the user's everyday life. }\end{array}$ \\
\hline $\begin{array}{l}\text { (Goyal et al., } \\
\text { 2016) }\end{array}$ & $\begin{array}{l}\text { The Knowledge to Action and Medical } \\
\text { Research Council provides a framework for } \\
\text { the development of intervention. }\end{array}$ & $\begin{array}{l}\text { Self-monitoring, providing actionable knowledge and } \\
\text { behavior change is aligned with the constructs of social } \\
\text { cognitive theory (SCT) }\end{array}$ \\
\hline $\begin{array}{l}\text { (Mayberry et } \\
\text { al., 2016) }\end{array}$ & $\begin{array}{l}\text { Intervention is developed through user- } \\
\text { centered design and iterative testing }\end{array}$ & Study employs techniques from Goal Setting Theory \\
\hline $\begin{array}{l}\text { (Mummah et } \\
\text { al., 2016) }\end{array}$ & $\begin{array}{l}\text { App development follows phases outlined by } \\
\text { the IDEAS framework. }\end{array}$ & $\begin{array}{l}\text { Study include } 18 \text { behavior change techniques (BCTs) } \\
\text { such as goal setting, feedback, social comparison, } \\
\text { prompts/cues. }\end{array}$ \\
\hline $\begin{array}{l}\text { (Gabrielli et } \\
\text { al., 2017) }\end{array}$ & $\begin{array}{l}\text { Design sessions is hold with a team of } \\
\text { Behavioral Change experts, working with } 2 \\
\text { nutritionists and } 3 \text { pediatricians }\end{array}$ & $\begin{array}{l}\text { Content is derived from knowledge founded on the Food } \\
\text { Pyramid and Mediterranean Diet Guidelines }\end{array}$ \\
\hline $\begin{array}{l}\text { (López et al., } \\
\text { 2017) }\end{array}$ & $\begin{array}{l}\text { App developed follows the ADDIE (analysis, } \\
\text { design, development, implementation, and } \\
\text { evaluation) model. }\end{array}$ & $\begin{array}{l}\text { List of healthy food is created based on Dietary } \\
\text { Guidelines for Americans }\end{array}$ \\
\hline $\begin{array}{l}\text { (Backman et } \\
\text { al., 2018) }\end{array}$ & $\begin{array}{l}\text { Study follows a user-centered design process } \\
\text { integrated with a modern agile software } \\
\text { development methodology to iteratively } \\
\text { codesign the app. }\end{array}$ & Paper-based Path to Home workbook. \\
\hline $\begin{array}{l}\text { (Direito et al., } \\
2018)\end{array}$ & $\begin{array}{l}\text { The intervention mapping (IM) framework } \\
\text { comprises six fundamental steps that guide the } \\
\text { design, implementation, and evaluation of an } \\
\text { intervention. }\end{array}$ & $\begin{array}{l}\text { Using the IM framework, study selects theoretical } \\
\text { constructs (e.g., perceived social norm, intention, and } \\
\text { competence) from the Integrated Behavior Change } \\
\text { Model (IBCM) and maps them to specific BCTs to } \\
\text { define intervention content. }\end{array}$ \\
\hline $\begin{array}{l}\text { (Giunti et al., } \\
\text { 2018) }\end{array}$ & $\begin{array}{l}\text { Study identifies needs, barriers, and } \\
\text { facilitators for the app for following UCD. }\end{array}$ & $\begin{array}{l}\text { Design decisions were evidence-driven and guided by } \\
\text { behavioral change models (BCM) }\end{array}$ \\
\hline
\end{tabular}

As the table indicates, mHealth studies with design-based and evidence-based methodology had only emerged after 2015. There are several implications of this summary. Using clinical guidelines is a concrete way to develop evidence-based mHealth interventions as long as they correspond to intervention requirements (Gabrielli et al., 2017; López et al., 2017; Jeon and Park, 2015). However, single BCT or clinical guideline may not suffice all the requirements for an mHealth intervention thus blended approaches can also be adopted (Lin et al., 2015; Revenäs et al., 2015; Mummah et al., 2016).

Furthermore, many mHealth researches are long-period efforts and requirements can change during that period. Users can receive the final product as archaic at the end of study or the mHealth application can fall short for satisfying recent requirements due to fast changing nature of mobile technologies. In order to keep pace with fast changing user needs and technologies, agile software development practices along with iterative and incremental design techniques can be preferred. In this regard, minimal viable product (MVP) 
and validated learning is good practices originated from the "Lean Startup" philosophy to eliminate wasteful practices by incorporating user feedback into early stage of development (Tregarthen et al., 2015).

Apparently, mHealth interventions are growing in technical complexity as well as in behavioral change techniques used within them. Therefore, it becomes essential to use specific frameworks, which obliges study to be evidence-based and to structure the design process in a systematic manner at the same time. MRC, DSR, IDEAS, and intervention mapping are some of favorable frameworks that is discovered within the literature review and recommended for future mHealth studies to guide their overall study.

\section{CONCLUSION}

This paper investigates methodological foundations of mHealth studies regarding the app design and content development processes and investigates the current situation in the field by reviewing articles published in last 10 years. Unlike the previous literature reviews, we emphasis understanding theoretical grounds and methodological rigor before understanding the success and failure reasons of mHealth interventions. The content analysis shows that even user-centered design (UCD) is a common approach for mHealth design studies, only few of them adopts a systematic approach (e.g. DSR Framework) to guide design process or content development. However, complex mHealth interventions requires both evidence-based and design-based approaches to go beyond initial adoption and cover socio-technical aspects of intervention such as scalability, long term engagement, financial sustainability. Therefore, lack of methodological rigor in mHealth design studies can be another factor to explain lack of scaled and sustainable mHealth interventions. This study investigates and quantitatively analyze theoretical approaches within mHealth studies and recommends utilization of frameworks tailored to mHealth intervention. Future studies can investigate not only the design, development but also evaluation methodologies of mHealth applications.

\section{REFERENCES}

Alharbi, I. et al. (2018) 'Big Data Based m-Health Application to Prevent Health Hazards: A Design Science Framework', Telemedicine and e-Health, p. tmj.2018.0063. doi: 10.1089/tmj.2018.0063.

AlJaberi, H. (2018) 'Social Interaction Needs and Entertainment Approaches to Pregnancy Well-Being in mHealth Technology Design for Low-Income Transmigrant Women: Qualitative Codesign Study', JMIR mHealth and uHealth, 6(4), p. e61. doi: 10.2196/mhealth.7708.

Alnosayan, N. et al. (2017) 'Design and Usability of a Heart Failure mHealth System: A Pilot Study', JMIR Human Factors, 4(1), p. e9. doi: 10.2196/humanfactors.6481.

Alvarado, M. M. et al. (2017) 'Barriers to Remote Health Interventions for Type 2 Diabetes: A Systematic Review and Proposed Classification Scheme', Journal of Medical Internet Research, 19(2), p. e28. doi: 10.2196/jmir.6382.

Backman, C. et al. (2018) 'Development of a Path to Home mobile application for the geriatric rehabilitation program at Bruyere Continuing Care: A protocol for user-centered design and feasibility testing studies (Preprint)', JMIR Research Protocols. doi: 10.2196/11031.

Choo, S. et al. (2016) 'Development of a Weight Loss Mobile App Linked With an Accelerometer for Use in the Clinic: Usability, Acceptability, and Early Testing of its Impact on the Patient-Doctor Relationship', JMIR mHealth and uHealth, 4(1), p. e24. doi: 10.2196/mhealth.4546.

Direito, A. et al. (2018) 'Using the Intervention Mapping and Behavioral Intervention Technology Frameworks: Development of an mHealth Intervention for Physical Activity and Sedentary Behavior Change', Health Education \& Behavior, 45(3), pp. 331-348. doi: 10.1177/1090198117742438.

Gabrielli, S. et al. (2017) 'Design of a Mobile App for Nutrition Education (TreC-LifeStyle) and Formative Evaluation With Families of Overweight Children', JMIR mHealth and uHealth, 5(4), p. e48. doi: 10.2196/mhealth.7080.

Gagnon, M.-P. et al. (2016) 'm-Health adoption by healthcare professionals: a systematic review', Journal of the American Medical Informatics Association, 23(1), pp. 212-220. doi: 10.1093/jamia/ocv052.

Giunti, G., Mylonopoulou, V. and Rivera Romero, O. (2018) 'More Stamina, a Gamified mHealth Solution for Persons with Multiple Sclerosis: Research Through Design', JMIR mHealth and uHealth, 6(3), p. e51. doi: $10.2196 /$ mhealth. 9437 .

Goyal, S. et al. (2016) 'The Systematic Design of a Behavioural Mobile Health Application for the Self-Management of Type 2 Diabetes', Canadian Journal of Diabetes, 40(1), pp. 95-104. doi: 10.1016/j.jcjd.2015.06.007.

Hamine, S. et al. (2015) 'Impact of mHealth chronic disease management on treatment adherence and patient outcomes: A systematic review', Journal of Medical Internet Research, 17(2), pp. 1-15. doi: 10.2196/jmir.3951. 
Ithnin, M. et al. (2017) 'Mobile App Design, Development, and Publication for Adverse Drug Reaction Assessments of Causality, Severity, and Preventability', JMIR mHealth and uHealth, 5(5), p. e78. doi: 10.2196/mhealth.6261.

Jeon, E. and Park, H.-A. (2015) 'Development of a Smartphone Application for Clinical-Guideline-Based Obesity Management', Healthcare Informatics Research, 21(1), p. 10. doi: 10.4258/hir.2015.21.1.10.

Jo, S. and Park, H.-A. (2016) 'Development and Evaluation of a Smartphone Application for Managing Gestational Diabetes Mellitus', Healthcare Informatics Research, 22(1), p. 11. doi: 10.4258/hir.2016.22.1.11.

Kim, K., Gollamudi, S. S. and Steinhubl, S. (2017) 'Digital technology to enable aging in place', Experimental Gerontology. Elsevier Inc., 88, pp. 25-31. doi: 10.1016/j.exger.2016.11.013.

Kruse, C. S. et al. (2018) 'Evaluating barriers to adopting telemedicine worldwide: A systematic review', Journal of Telemedicine and Telecare, 24(1), pp. 4-12. doi: 10.1177/1357633X16674087.

Laidlaw, R. et al. (2017) 'Using participatory methods to design an mHealth intervention for a low income country, a case study in Chikwawa, Malawi', BMC Medical Informatics and Decision Making, 17(1), p. 98. doi: 10.1186/s12911-017-0485-6.

Lau, Y. et al. (2018) 'Development of a Healthy Lifestyle Mobile App for Overweight Pregnant Women: Qualitative Study', JMIR mHealth and uHealth, 6(4), p. e91. doi: 10.2196/mhealth.9718.

Lin, P.-H. et al. (2015) 'Adaptive intervention design in mobile health: Intervention design and development in the Cell Phone Intervention for You trial', Clinical Trials: Journal of the Society for Clinical Trials, 12(6), pp. 634-645. doi: $10.1177 / 1740774515597222$.

López, D. et al. (2017) 'Development and Evaluation of a Nutritional Smartphone Application for Making Smart and Healthy Choices in Grocery Shopping', Healthcare Informatics Research, 23(1), p. 16. doi: 10.4258/hir.2017.23.1.16.

Lubans, D. R. et al. (2014) 'Development and Implementation of a Smartphone Application to Promote Physical Activity and Reduce Screen-Time in Adolescent Boys', Frontiers in Public Health, 2. doi: 10.3389/fpubh.2014.00042.

Matthew-Maich, N. et al. (2016) 'Designing, Implementing, and Evaluating Mobile Health Technologies for Managing Chronic Conditions in Older Adults: A Scoping Review', JMIR mHealth and uHealth, 4(2), p. e29. doi: $10.2196 /$ mhealth.5127.

Mayberry, L. S. et al. (2016) 'The Design, Usability, and Feasibility of a Family-Focused Diabetes Self-Care Support mHealth Intervention for Diverse, Low-Income Adults with Type 2 Diabetes', Journal of Diabetes Research, 2016, pp. 1-13. doi: 10.1155/2016/7586385.

Mehmood, A. et al. (2017) 'Development of an mHealth trauma registry in the Middle East using an implementation science framework', Global Health Action, 10(1), p. 1380360. doi: 10.1080/16549716.2017.1380360.

Moher, D. (2009) 'Preferred Reporting Items for Systematic Reviews and Meta-Analyses: The PRISMA Statement', Annals of Internal Medicine, 151(4), p. 264. doi: 10.7326/0003-4819-151-4-200908180-00135.

Mummah, S. A. et al. (2016) 'Iterative development of Vegethon: a theory-based mobile app intervention to increase vegetable consumption', International Journal of Behavioral Nutrition and Physical Activity, 13(1), p. 90. doi: 10.1186/s12966-016-0400-z.

Pereira-Azevedo, N. et al. (2017) 'Rotterdam Prostate Cancer Risk Calculator: Development and Usability Testing of the Mobile Phone App', JMIR Cancer, 3(1), p. e1. doi: 10.2196/cancer.6750.

Ravn Jakobsen, P. et al. (2018) 'Development of an mHealth Application for Women Newly Diagnosed with Osteoporosis without Preceding Fractures: A Participatory Design Approach', International Journal of Environmental Research and Public Health, 15(2), p. 330. doi: 10.3390/ijerph15020330.

Revenäs, ̊. et al. (2015) 'Development of a Web-Based and Mobile App to Support Physical Activity in Individuals With Rheumatoid Arthritis: Results From the Second Step of a Co-Design Process', JMIR Research Protocols, 4(1), p. e22. doi: 10.2196/resprot.3795.

Schnall, R. et al. (2016) 'A user-centered model for designing consumer mobile health (mHealth) applications (apps)', Journal of Biomedical Informatics, 60, pp. 243-251. doi: 10.1016/j.jbi.2016.02.002.

Steinhubl, S. R., Muse, E. D. and Topol, E. J. (2015) 'The emerging field of mobile health', Science Translational Medicine, 7(283), p. 283rv3-283rv3. doi: 10.1126/scitranslmed.aaa3487.

Thinnukool, O., Khuwuthyakorn, P. and Wientong, P. (2017) 'Pharmacy Assistant Mobile Application (PAMA): Development and Reviews', International Journal of Interactive Mobile Technologies (iJIM), 11(3), p. 178. doi: 10.3991/ijim.v11i3.6757.

Tregarthen, J. P., Lock, J. and Darcy, A. M. (2015) 'Development of a smartphone application for eating disorder self-monitoring', International Journal of Eating Disorders, 48(7), pp. 972-982. doi: 10.1002/eat.22386.

Vorrink, S. N. et al. (2016) 'A Mobile Phone App to Stimulate Daily Physical Activity in Patients with Chronic Obstructive Pulmonary Disease: Development, Feasibility, and Pilot Studies', JMIR mHealth and uHealth, 4(1), p. e11. doi: 10.2196/mhealth.4741.

Zhu, J. et al. (2017) 'Development of a mobile application of Breast Cancer e-Support program for women with breast cancer undergoing chemotherapy', Technology and Health Care, 25(2), pp. 377-382. doi: 10.3233/THC-161292. 